\title{
Lactate Control Enhances Growth Advantage in Fed-batch Cultures of Metabolically Engineered CHO Cells with Reduced Novel Growth-inhibitory Compound Formation
}

\author{
Quentin Bethune $^{1}$, Cameron Harrington ${ }^{1}$, and Bhanu Mulukutla ${ }^{1}$ \\ ${ }^{1}$ Pfizer Andover
}

January 18, 2021

\begin{abstract}
CHO cells have been recently shown to produce amino acid catabolism derived byproducts, which accumulate in fed-batch cultures to growth-inhibitory levels. Residual amino acid limitation or genetic engineering strategies have been successfully employed to suppress production of these novel growth inhibitory metabolic byproducts. However, the growth advantage attained due to suppression of these metabolic byproducts in fed-batch cultures is more pronounced when lactate accumulation is also controlled. BCAT1 knock-out (KO) CHO cells, which produce negligible levels of the metabolic byproducts isovalerate, isobutyrate and 2-methylbutyrate, grow to significantly higher peak cell densities in fed-batch cultures with lactate control (HiPDOG) as compared to cultures without lactate control. Henceforth, strategies involving novel metabolic byproduct control should preferably include lactate control to more easily assess the enhanced cell growth and productivities attainable.

Communication to Editor

Lactate Control Enhances Growth Advantage in Fed-batch Cultures of Metabolically Engineered CHO Cells with Reduced Novel Growth-inhibitory Compound Formation

Quentin Bethune $^{1}$, Cameron Harrington ${ }^{1}$ and Bhanu Chandra Mulukutla ${ }^{2}$

Upstream Process Development, Pfizer Inc, One Burtt Road, Andover MA, 01810, USA

1 Contributed equally

2 Corresponding author (email: bhanuchandra.mulukutla@pfizer.com)
\end{abstract}

\section{Abstract}

CHO cells have been recently shown to produce amino acid catabolism derived byproducts, which accumulate in fed-batch cultures to growth-inhibitory levels. Residual amino acid limitation or genetic engineering strategies have been successfully employed to suppress production of these novel growth inhibitory metabolic byproducts. However, the growth advantage attained due to suppression of these metabolic byproducts in fed-batch cultures is more pronounced when lactate accumulation is also controlled. BCAT1 knock-out (KO) CHO cells, which produce negligible levels of the metabolic byproducts isovalerate, isobutyrate and 2-methylbutyrate, grow to significantly higher peak cell densities in fed-batch cultures with lactate control (HiPDOG) as compared to cultures without lactate control. Henceforth, strategies involving novel metabolic byproduct control should preferably include lactate control to more easily assess the enhanced cell growth and productivities attainable. 
Keywords: Fed-Batch Cell Culture, CHO, Lactate Control, Growth Inhibition, Metabolic Byproducts, Metabolic Engineering

\section{Introduction}

CHO cells are currently the workhorse for therapeutic protein production of various therapeutic modalities including monoclonal antibodies, antibody drug conjugates, enzymes, vaccines, etc. Over the last decade, fed-batch has been the predominant culture mode for protein production using CHO cells. While titers for $\mathrm{mAb}$ production in fed-batch cultures have significantly increased over this time span, currently commonly reaching $5-10 \mathrm{~g} / \mathrm{L}$, that productivity progress may have reached a ceiling. To add to this, the advent of the biosimilars has created an additional driver for cost of goods reduction and an increase in process productivities.

To push the productivities higher, perfusion or hybrids of perfusion and fed-batch processes are currently being explored (Hiller et al., 2017). The underlying rational for such a move towards perfusion processes is the building consensus that productivity is principally being limited due to the accumulation of growthinhibitory metabolic byproducts (Pereira et al., 2018; Mulukutla et al., 2017). Flushing such inhibitors out of the culture using perfusion is a straightforward way of improving the culture environment and boosting cellular proliferation (Hiller et al., 2017). However, the introduction of perfusion does present manufacturing challenges in the form of operational and facility fit complexities. Fed-batch processes are still a preferred way of manufacturing biologics due to manufacturing ease they present. Therefore, improving titers and productivities in fed-batch cultures continues to be very desirable.

Lactate and ammonia are the classical metabolic byproducts that are known to accumulate in fed-batch cultures. It has been extensively documented in the published literature that the aforementioned byproducts inhibit growth and productivities to varying degrees at high concentrations (Lao and Toth, 1997; Cruz et al., 2000; Hansen and Emborg, 1994; Kurano et al., 1990; Ozturk et al., 1992) and significant research efforts have been devoted towards their control in culture (O'Brien et al., 2020). In the case of lactate, process and genetic engineering methods have been employed to reduce its formation, some of which have been reviewed recently (Hartley et al., 2018; Kim and Lee, 2007; Zhou et al., 2011; Toussaint et al., 2016). The use of HiPDOG technology to control lactate accumulation by limiting residual glucose levels in culture has shown successful results (Gagnon et al., 2011). HiPDOG technology uses culture pH as an indirect measure of glucose to maintain residual culture glucose levels in a low concentration range. This results in net reduced lactate accumulation in culture, thereby allowing cells to grow to higher densities which yield higher titers. However, even in such cultures cells eventually stop proliferating due to inhibitory action of other byproducts which accumulate throughout the culture (Mulukutla et al., 2017).

More recently, the cell culture community has been investing in understanding metabolic byproducts beyond lactate and ammonia that accumulate in CHO cell fed-batch cultures (Pereira et al., 2018). We and others have shown that $\mathrm{CHO}$ cells produce metabolic byproducts from amino acid catabolism and glucose metabolism (Mulukutla et al., 2017; Alden et al., 2020). These byproducts can accumulate to growthinhibitory levels in $\mathrm{CHO}$ cell fed-batch cultures. We have reported that reducing accumulations of these inhibitory byproducts by maintaining levels of the corresponding amino acids within a low concentration range, under conditions of reduced lactate accumulation (using HiPDOG control), results in higher peak cell densities and productivities (Mulukutla et al., 2017). Alternatively, we have shown that genetic engineering approaches can be employed to overexpress certain enzymes, as in the Phe-Tyr pathway, to direct flux away from byproduct production. Additionally, knockout (KO) of enzymes, as in case of the branched-chain amino acid (BCAA) catabolic pathway, can be used to eliminate overflow metabolism and byproduct formation (Mulukutla et al., 2019). In the BCAA pathway, we have shown that KO of the BCAT1 gene, encoding for branched chain amino acid aminotransferase 1 enzyme, eliminates production of three growth-inhibitory byproducts: isovalerate, 2-methylbutyrate and isobutyrate. These inhibitors are catabolic byproducts of the amino acids, leucine, isoleucine and valine, respectively. The KO clones when grown in conditions with 
lactate control yield higher peak cell densities and productivities. Other groups have replicated this work, subsequently (Pereira et al., 2019). However, the impact of lactate control on such metabolically engineered cells hasn't been carefully evaluated yet.

In the present study, the benefit of controlling lactate production by employing the HiPDOG glucose limitation strategy on growth and productivity of BCAT1 KO cells was evaluated. In fed-batch cultures without control of lactate due, BCAT1 KO clones achieved, to a smaller degree, higher peak viable cell densities and titers when compared with their wild-type counterparts. However, in glucose-limited HiPDOG cultures BCAT1 KO cells grew to significantly higher cell densities and produced higher titers than the wild-type cells.

\section{Materials and Methods}

\section{Cells, medium and shake-flask cultures}

Three clones derived from CHO Cell Line B (Wild-type Clone 47, BCAT1 KO Clone 83, and BCAT1 KO Clone 90) utilizing a glutamine synthetase expression system and expressing a recombinant antibody were used in the present study (Mulukutla et al., 2019). Three types of proprietary medium were used: "Medium A" is the production medium for traditional and HiPDOG fed-batch production bioreactors. "Medium B" is an enriched nutrient solution used as feed medium for both modes of production bioreactor. Medium $\mathrm{C}$ is the passaging medium used for cultivation and experimentation in shake-flask batch cultures. Medium A and B are identical in composition to those used previously (Mulukutla et al., 2019).

Medium A is a chemically defined, protein-free, amino acid fortified version of DMEM:F12 medium with adjusted levels of vitamins, trace elements, sodium bicarbonate and potassium chloride, also containing polyvinyl alcohol. Medium A was modified with additional sodium bicarbonate and glucose for non-HiPDOG fed-batch production bioreactors. Medium B is a chemically defined, protein-free concentrated feed composed of amino acids, vitamins, and trace elements (a subset of those in Medium A). Medium B was supplemented with additional alanine, cysteine, tyrosine, and zinc to prevent depletion of these compounds in high-density cell culture processes.

The specific impact of lactate on cell growth of BCAT1 KO Clone 83 and WT Clone 47 was assessed using shake-flask cultures. Medium C, a chemically defined and protein-free lean passaging medium was modified with 0 to $10 \mathrm{~g} / \mathrm{L}$ lactate via sodium lactate (Sigma-Aldrich@, St. Louis, MO) at concentrations similar to those achieved in fed-batch and HiPDOG bioreactors. An osmolality control matching the $8 \mathrm{~g} / \mathrm{L}$ lactate condition was also made using Medium $\mathrm{C}$ modified with sodium chloride. WT Clone 47 sodium lactate concentrations used were a subset of those used for Clone 83, included for comparability. Cells were inoculated at $0.1 \times 10^{6}$ cells $/ \mathrm{mL}$ in triplicate $125 \mathrm{~mL}$ shake-flasks for each condition with a working volume of $30 \mathrm{~mL}$, and cultivated for 6 days on a shaking platform (Orbital Shaker, Bellco Glass, Inc.) in a humidified incubator (ThermoFisher Scientific) maintained at $36.5 \mathrm{degC}$ and $5 \%$ carbon dioxide. Viable cell density, glucose, lactate, and ammonia concentrations in the cell culture medium were measured on days 0,3 , and 6 using a Nova Bioprofile FLEX Analyzer (Nova Biomedical, Waltham, USA).

\section{Bioreactor setup for fed-batch and HiPDOG cultures}

Cell Line B clones were cultivated in pairs of bioreactors where the first of each pair employed a typical titrant-based lower pH dead-band and the second, the HiPDOG control strategy (Gagnon et al., 2011). For HiPDOG bioreactors, $\mathrm{pH}$ dead-bands during and after HiPDOG were 7.125 +/- 0.025 and $7.10+/-0.20$ respectively. Each fed-batch bioreactor had its pH tightly controlled to match its HiPDOG partner during the HiPDOG operational phase, while utilizing the same $\mathrm{pH}$ dead-band thereafter. Medium A was used as the production medium, while Medium B was used as feed medium for all vessels. Inoculation cell densities were $1.5 \times 10^{6}$ cells $/ \mathrm{mL}$ in an initial working volume of $1 \mathrm{~L}$, with temperature and agitation set to $36.5^{\circ} \mathrm{C}$ 
and $259 \mathrm{rpm}$ respectively. In HiPDOG reactors, feed medium was added based on upper $\mathrm{pH}$ dead-band during HiPDOG control, operational from day 2 to day 6 . During this period each fed-batch reactor had constant semi-continuous feed rates set daily to match the cell specific feed rate of its partner HiPDOG reactor. Fed-batch reactors were additionally provided glucose to target $2.5 \mathrm{~g} / \mathrm{L}$ as-needed. Following the end of HiPDOG control, feed rates for all vessels were set daily to target a final cumulative cell specific feed rate of $1.75 \mathrm{pL} /$ cell/day, resulting in a total feed medium addition of 20 to $60 \%$ of the culture starting volume. Post-HiPDOG glucose levels were targeted at $2.5 \mathrm{~g} / \mathrm{L}$ by feeding additional glucose as necessary for all vessels. Viable cell density, glucose, lactate, and ammonia concentrations in the cell culture medium were measured daily using a Nova Bioprofile FLEX Analyzer (Nova Biomedical, Waltham, USA). Titer analysis was performed by Protein A HPLC (model 1100 HPLC, Agilent Technologies, Inc., Santa Clara, CA, protein A column model 2-1001-00, Applied Biosystems, Foster City, CA). WT clone 47 conditions were run in duplicate, however data beyond day 11 for the duplicate HiPDOG condition are unavailable due to a controlling probe failure.

Additional methods pertaining to amino acid concentration analysis via UPLC and spent medium metabolite analysis via NMR are identical to those utilized in the prior study (Mulukutla et al., 2019).

\section{Results and Discussion}

CHO cells produce the growth-inhibitory metabolites isovalerate, isobutyrate and 2-methylbutyrate through catabolism of leucine, isoleucine and valine. Previously, we have reported the generation of CHO cell clones with KO of the BCAT1 gene from an antibody producing wildtype (WT) cell line, Cell Line B (Mulukutla et al., 2019). The names of these clones are BCAT1 KO Clone 83, 86 and 90. To probe the impact of lactate control on performance of BCAT1 KO clones, Clone 83, Clone 90 and WT cells were cultivated in fed-batch cultures and HiPDOG cultures. All process parameters were kept the same between fed-batch and HiPDOG conditions, except for residual glucose levels (see Materials and Methods). Nutrient feed was delivered such that amount of feed added on a per cell basis was relatively constant across all conditions.

In non-HiPDOG fed-batch cultures, residual glucose levels were maintained above $2 \mathrm{~g} / \mathrm{L}$ throughout the culture with the exception of day 3, where glucose levels fell to $1 \mathrm{~g} / \mathrm{L}$ in WT conditions (Figure 1). The BCAT1 KO clones (83 and 90) had comparable growth rates to the WT in the initial few days of the culture but achieved $3-7 \times 10^{6}$ cells/mL higher peak cell densities than the WT cells. Viability profiles were similar across all conditions. Lactate profiles were also similar across the conditions, with WT cultures peaking slightly higher than BCAT1 KO cultures. The lactate peak across all conditions was within a relatively high concentration range $(6.5-8 \mathrm{~g} / \mathrm{L})$, but a good metabolic shift to lactate consumption was observed across all conditions. Notably, the BCAT1 KO cultures had higher titers than the WT, attributable to higher specific productivity $\left(q_{P}\right)$ in the BCAT1 KO cultures when compared with the WT cultures.

For HiPDOG cultures, the HiPDOG control strategy was operational from day 2 to 6 , during which residual glucose levels were maintained in the $0.01-0.2 \mathrm{~g} / \mathrm{L}$ range. After ending the HiPDOG control strategy, residual glucose levels were maintained above $2 \mathrm{~g} / \mathrm{L}$ (Figure 2). BCAT1 KO clones (83 and 90) and WT cells had very similar growth rates for the initial four days of the culture. Subsequently WT growth rates slowed, and these cultures peaked at roughly $21 \times 10^{6}$ cells $/ \mathrm{mL}$ on day 9 . However, BCAT1 KO clones continued to grow at high growth rates, reaching peak VCD on day 10 of $44 \times 10^{6}$ and $50 \times 10^{6}$ cells/mL; about $23-30$ x $10^{6}$ cells $/ \mathrm{mL}$ higher peak VCD than WT counterparts. Severe foaming in BCAT1 KO cultures may have limited the peak cell densities. Lactate was well-controlled across all conditions, with peak concentrations around $3 \mathrm{~g} / \mathrm{L}$ and good metabolic shift to lactate consumption. BCAT1 KO cultures also had significantly higher titers than the WT counter parts, which can be attributed to higher peak VCDs as the $q_{P}$ was similar across all the conditions.

Levels of the metabolic byproducts isovalerate, isobutyrate and 2-methylbutyrate are negligible across fedbatch and HiPDOG conditions for BCAT1 KO clones (Figure 1, Figure 2), which is expected given the 
encoded enzyme's role as the entry point for BCAAs into the catabolic pathways responsible for production of these compounds (Mulukutla et al., 2019). Accumulation of these byproducts was observed in all the wildtype cultures, albeit to different levels across fed-batch and HiPDOG conditions. The cause of differences in byproduct accumulation between fed-batch and HIPDOG cultures will be investigated in a future study.

The levels of other previously reported growth-inhibitory metabolic byproducts were probed in the culture milieu of HiPDOG and fed-batch cultures of BCAT1 KO and WT clones on days 6, 8 and 10 (Figure 3) (Mulukutla et al., 2017). 3-phenyllactate and 4-hydroxyphenyllactate levels were similar between HiPDOG and fed-batch cultures until day 6 but increased over time in the HiPDOG cultures. Indole-3-lactate levels were higher in HiPDOG cultures across all the time points probed. Butyrate levels were similar between HiPDOG and fed-batch cultures across all the time points probed. Formate and glycerol levels were similar between HiPDOG and fed-batch cultures on day 6 but accumulated to lower levels in HiPDOG cultures thereafter. Concentrations of all tested metabolic byproducts were very similar between HiPDOG and fedbatch cultures on day 6 , thus the reduced growth observed in fed-batch BCAT1 KO cultures by day 6 can be concluded not to be due to accumulation of these other metabolic byproducts in the fed-batch culture milieu.

Previously, it has been reported that fed-batch cultures with robust metabolic shifts perform better than those without (Le et al., 2012). The above data suggests even though the BCAT1 KO clone can undergo a strong metabolic shift in fed-batch cultures, controlling peak lactate levels, as in the case of HiPDOG cultures, appears to greatly increase peak cell densities. The higher peak lactate and corresponding higher osmolality observed in standard fed-batch cultures could be negatively impacting the proliferative capability of BCAT1 KO cells. This hypothesis was put to test in a subsequent experiment. The BCAT1 KO Clone 83 was inoculated in shake flask cultures at $0.1 \times 10^{6}$ cells $/ \mathrm{mL}$ and treated with different levels of lactate spanning the levels observed in the fed-batch and HiPDOG conditions (Figure 4). Growth characteristics of all conditions were probed over the course of 6 days. An osmolality control was also included, targeting the $8 \mathrm{~g} / \mathrm{L}$ lactate condition. BCAT1 KO clone growth was similar across 0 and $2.5 \mathrm{~g} / \mathrm{L}$ conditions between days 0 and 3, with slightly reduced growth rate for the remainder of the batch. Substantial growth inhibition was observed at and above the $5 \mathrm{~g} / \mathrm{L}$ condition. The osmolality control also had significantly lower growth when compared with the $0 \mathrm{~g} / \mathrm{L}$ condition. When WT cells in shake-flask cultures were subjected to similar lactate concentrations, similar growth inhibition to that of BCAT1 KO clone cultures was observed. These data suggest that higher peak lactate levels and the associated osmolality could have a negative effect on the growth of $\mathrm{CHO}$ cells in fed-batch cultures even if they undergo a robust metabolic shift. The above result that lactate suppresses growth rate is not novel. This has been reported widely in the past across multiple mammalian cell lines used for therapeutic production, including $\mathrm{CHO}$, hybridoma, baby hamster kidney (BHK) cells etc. (Cruz et al., 2000; Lao and Toth, 1997; Kurano et al., 1990; Ozturk et al., 1992).

Control of residual glucose at low concentrations could also have a positive effect on growth of the BCAT1 $\mathrm{KO}$ cells, independent of lactate accumulation in fed-batch cultures. Previous studies with hybridoma cells have shown that exposure to lower residual glucose levels for prolonged amount of time in batch or fedbatch mode before transition into continuous culture mode can result in a lower ratio of lactate produced to glucose consumed during the continuous phase of the culture (Europa et al., 2000). Expression of certain key glycolytic genes were downregulated in culture with exposure to lower glucose concentration (Korke et al., 2004). Whether any such changes at the transcriptome level due to reduced exposure to glucose in HiPDOG cultures have a role to play in improved performance of BCAT1 KO cells remains to be ascertained.

In summary, metabolic engineering towards reduction of novel growth inhibitors can help increase cell densities and titers of fed-batch cultures. However, close attention needs to be paid to the level of lactate accumulation as lactate is the major growth-inhibitory metabolic byproduct produced by CHO cells in culture. Accumulation of lactate to very high levels can reduce the proliferative advantage offered by control of other inhibitory metabolic byproducts in fed-batch cultures. Future metabolic engineering attempts that can simultaneously eliminate production of lactate and BCAA metabolic products including isovalerate, isobutyrate and 2-methylbutyrate can result in fed-batch culture cell growth rivaling that of perfusion cultures. 


\section{Acknowledgements}

The authors would like to acknowledge Samuel Perakis for assistance with HPLC methods.

\section{References}

Alden N, Raju R, McElearney K, Lambropoulos J, Kshirsagar R, Gilbert A, Lee K. 2020. Using Metabolomics to Identify Cell Line-Independent Indicators of Growth Inhibition for Chinese Hamster Ovary Cell-Based Bioprocesses. Metabolites 10:199.

Cruz HJ, Freitas CM, Alves PM, Moreira JL, Carrondo MJT. 2000. Effects of ammonia and lactate on growth, metabolism, and productivity of BHK cells. Enzyme and Microbial Technology 27:43-52.

Europa AF, Gambhir A, Fu P-C, Hu W-S. 2000. Multiple steady states with distinct cellular metabolism in continuous culture of mammalian cells. Biotechnology and Bioengineering 67:25-34.

Gagnon M, Hiller G, Luan Y-T, Kittredge A, DeFelice J, Drapeau D. 2011. High-End pH-controlled delivery of glucose effectively suppresses lactate accumulation in CHO Fed-batch cultures. Biotechnology and Bioengineering 108:1328-1337.

Hansen HA, Emborg C. 1994. Influence of Ammonium on Growth, Metabolism, and Productivity of a Continuous Suspension Chinese Hamster Ovary Cell Culture. Biotechnology Progress 10:121-124.

Hartley F, Walker T, Chung V, Morten K. 2018. Mechanisms driving the lactate switch in Chinese hamster ovary cells. Biotechnology and Bioengineering 115:1890-1903.

Hiller GW, Ovalle AM, Gagnon MP, Curran ML, Wang W. 2017. Cell-controlled hybrid perfusion fedbatch $\mathrm{CHO}$ cell process provides significant productivity improvement over conventional fed-batch cultures. Biotechnology and Bioengineering 114:1438-1447.

Kim SH, Lee GM. 2007. Down-regulation of lactate dehydrogenase-A by siRNAs for reduced lactic acid formation of Chinese hamster ovary cells producing thrombopoietin. Appl Microbiol Biotechnol 74:152-159.

Korke R, Gatti M de L, Lau ALY, Lim JWE, Seow TK, Chung MCM, Hu W-S. 2004. Large scale gene expression profiling of metabolic shift of mammalian cells in culture. Journal of Biotechnology 107:1-17.

Kurano N, Leist C, Messi F, Kurano S, Fiechter A. 1990. Growth behavior of Chinese hamster ovary cells in a compact loop bioreactor. 2. Effects of medium components and waste products. Journal of Biotechnology 15. Mammalian Cell Structure:113-128.

Lao M-S, Toth D. 1997. Effects of Ammonium and Lactate on Growth and Metabolism of a Recombinant Chinese Hamster Ovary Cell Culture. Biotechnology Progress 13:688-691.

Le H, Kabbur S, Pollastrini L, Sun Z, Mills K, Johnson K, Karypis G, Hu W-S. 2012. Multivariate analysis of cell culture bioprocess data - Lactate consumption as process indicator. Journal of Biotechnology 162:210223 .

Mulukutla BC, Kale J, Kalomeris T, Jacobs M, Hiller GW. 2017. Identification and control of novel growth inhibitors in fed-batch cultures of Chinese hamster ovary cells. Biotechnology and Bioengineering 114:17791790 .

Mulukutla BC, Mitchell J, Geoffroy P, Harrington C, Krishnan M, Kalomeris T, Morris C, Zhang L, Pegman P, Hiller GW. 2019. Metabolic engineering of Chinese hamster ovary cells towards reduced biosynthesis and accumulation of novel growth inhibitors in fed-batch cultures. Metabolic Engineering 54:54-68.

O'Brien CM, Mulukutla BC, Mashek DG, Hu W-S. 2020. Regulation of Metabolic Homeostasis in Cell Culture Bioprocesses. Trends in Biotechnology 38. Special Issue: Therapeutic Biomanufacturing:1113-1127. 
Ozturk SS, Riley MR, Palsson BO. 1992. Effects of ammonia and lactate on hybridoma growth, metabolism, and antibody production. Biotechnology and Bioengineering 39:418-431.

Pereira S, Kildegaard HF, Andersen MR. 2018. Impact of CHO Metabolism on Cell Growth and Protein Production: An Overview of Toxic and Inhibiting Metabolites and Nutrients. Biotechnology Journal $13: 1700499$.

Pereira S, Ley D, Schubert M, Grav LM, Kildegaard HF, Andersen MR. 2019. BCAT1 and BCAT2 disruption in CHO cells has cell line-dependent effects. Journal of Biotechnology 306:24-31.

Toussaint C, Henry O, Durocher Y. 2016. Metabolic engineering of CHO cells to alter lactate metabolism during fed-batch cultures. Journal of Biotechnology 217:122-131.

Zhou M, Crawford Y, Ng D, Tung J, Pynn AFJ, Meier A, Yuk IH, Vijayasankaran N, Leach K, Joly J, Snedecor B, Shen A. 2011. Decreasing lactate level and increasing antibody production in Chinese Hamster Ovary cells $(\mathrm{CHO})$ by reducing the expression of lactate dehydrogenase and pyruvate dehydrogenase kinases. Journal of Biotechnology 153:27-34.

\section{Figure Legends}

Figure 1: Growth and metabolic characteristics of WT and BCAT1 KO clones in fed-batch cultures. A.) Viable Cell Density, B.) Viability, C.) Lactate, D.) Glucose, E.) Titer, F.) Specific Productivity, G.) isovalerate H.) isobutyrate, I.) 2-methylbutyrate. BCAT1 KO Clone 83 (), BCAT KO Clone 90 ( ), WT Clone 47 ( ). For WT Clone 47, solid and dotted lines represent duplicates.

Figure 2: Growth and metabolic characteristics of WT and BCAT1 KO clones in HiPDOG cultures. A.) Viable Cell Density, B.) Viability, C.) Lactate, D.) Glucose, E.) Titer, F.) Specific Productivity, G.) isovalerate H.) isobutyrate, I.) 2-methylbutyrate. BCAT1 KO Clone 83 (), BCAT KO Clone 90 ( ), WT Clone 47 ( ). For WT Clone 47, solid and dotted lines represent duplicates.

Figure 3: Levels of other metabolic byproducts across fed-batch and HiPDOG cultures of BCAT1 KO clones. A.) 3-phenyllactate, B.) 4-hydroxyphenyllactate, C.) indole-3-lactate, D.) butyrate, E.) formate and F.) glycerol concentrations in fed-batch and HiPDOG cultures of BCAT1 KO Clone 83 () and BCAT1 KO Clone 90 ( ). Solid and dotted lines represent fed-batch and HiPDOG cultures, respectively.

Figure 4: Effect of lactate on growth of CHO shake-flask batch cultures. A.) BCAT1 KO Clone 83 and B.) WT Clone 47 viable cell density over 6 days at added lactate concentrations ranging from 0 to 10 $\mathrm{g} / \mathrm{L}$. Lactate concentrations gradually increased by roughly $2 \mathrm{~g} / \mathrm{L}$ during the culture for control, osmolality control and $2.5 \mathrm{~g} / \mathrm{L}$ lactate conditions but did not change substantially for higher concentrations (data not shown). All conditions were run in triplicate. Error bars represent one standard deviation.

\section{Hosted file}

Figures_13Dec20.pdf available at https://authorea.com/users/390258/articles/504652-lactatecontrol-enhances-growth-advantage-in-fed-batch-cultures-of-metabolically-engineered-chocells-with-reduced-novel-growth-inhibitory-compound-formation 